This item was submitted to Loughborough's Research Repository by the author.

Items in Figshare are protected by copyright, with all rights reserved, unless otherwise indicated.

\title{
A novel current density distribution sensorfor use in parallel plate transmission lines
}

PLEASE CITE THE PUBLISHED VERSION

http://dx.doi.org/10.1109/EML.2014.6920696

PUBLISHER

IEEE

VERSION

AM (Accepted Manuscript)

\section{PUBLISHER STATEMENT}

This work is made available according to the conditions of the Creative Commons Attribution-NonCommercialNoDerivatives 4.0 International (CC BY-NC-ND 4.0) licence. Full details of this licence are available at: https://creativecommons.org/licenses/by-nc-nd/4.0/

\section{LICENCE}

CC BY-NC-ND 4.0

\section{REPOSITORY RECORD}

Omar, Kaashif A.M., N. Graneau, Bucur M. Novac, and Ivor R. Smith. 2019. "A Novel Current Density Distribution Sensorfor Use in Parallel Plate Transmission Lines”. figshare. https://hdl.handle.net/2134/20462. 


\title{
A Novel Current Density Distribution Sensor For Use In Parallel Plate Transmission Lines
}

\author{
K. Omar ${ }^{1,2}$, N. Graneau ${ }^{1}$, B. Novac ${ }^{2}$ and I.Smith ${ }^{2}$ \\ ${ }^{1}$ Hydrodynamics Department, AWE, Aldermaston, Reading, RG7 4PR, UK \\ ${ }^{2}$ School of Electronic, Electrical and Systems Engineering, Loughborough University, \\ Loughborough, Leicestershire LE11 3UE, UK
}

\begin{abstract}
As part of an on-going electromagnetic flyer plate research programme, it has been established that a method to determine the current distribution (or at least confirm its uniformity) is now required to establish predictable acceleration of the flyer plate. To achieve this diagnostic capability, a bespoke sensor array has been developed, termed the MIDOT (a relative Mutual Inductance sensor array). It has been specifically developed as a method of determining the current density distribution across a thin, close coupled, parallel plate transmission line.

This novel sensor has been developed to a point where the proof of principle has been successfully demonstrated on a low voltage test bed, with some preliminary high voltage shots being carried out on a bespoke experimental arrangement prior to it being fielded on the flyer plate accelerator facilities at both Loughborough University and AWE.
\end{abstract}

\section{INTRODUCTION}

Following on from earlier work [1] this paper focusses on the development of the MIDOT sensor and its use in bespoke low and high voltage installations. Both the one and two dimensional computer models developed [2] are utilised to understand and also plan the MIDOT experiments.

\section{PROCEDURE FOR PAPER SUBMISSION}

\section{A. Overview}

As stated in earlier work [2] current flowing in wide transmission lines cannot always be assumed to be uniformly distributed across their cross sections. The $2 \mathrm{D}$ filamentary model has been used to predict this non-uniform current distribution in the past [2] as well as the filamentary current distribution in various other geometries [3].

The MIDOT can be used to validate the model experimentally and get a direct measurement of the variation in the current distribution.

The sensor proposed here is termed "MIDOT", as it is related to the mutual inductance $(\mathrm{M})$ and the rate of change of current (dI/dt or 'I-Dot') in the circuit ('MIdot'). The 2D model assumes the sensor cross section to be equal to the cross section of the filaments used to divide the conductor as seen in Figure 1. This allows the mutual coupling to be calculated accurately.

To analyse the results, either the induced "open-circuit" voltages (Mdi/dt) from the sensors are then integrated and compared to the theoretically predicted values or the signals from an array of the probes are compared. Any variation between adjacent MIDOT probes indicates a non-uniform current distribution flowing below the sensors.

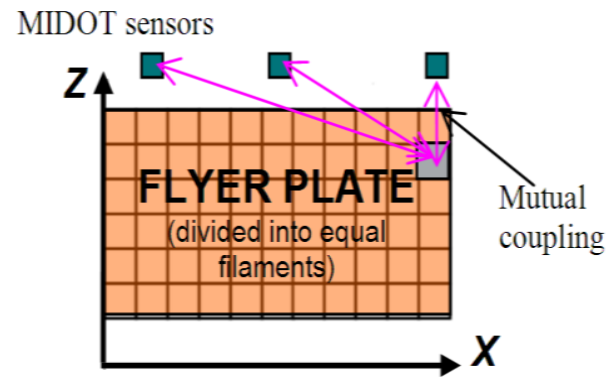

Figure 1, Proposed array of MIDOT sensors installed above the flyer plate conductor, which is divided into filaments to allow for the application of the $2 \mathrm{D}$ model [2].

Initial tests were limited to conductors which were only one filament thick, making for a simpler application of the model.

\section{B. Experimental arrangement for testing MIDOT}

The initial experiments focussed on taking readings due to current flowing in a single thin conductor (a filament). This work has been described in detail in a previous paper [4].

The work in this paper focusses on the signal due to a pseudo-stripline; comprised of individually insulated filaments in which the go and return conductors are shorted at one end and separated by $2 \mathrm{~mm}$ of polyethylene (Figure 2.).

The sensor array is positioned away from the ends of the stripline to ensure they do not affect the results. 


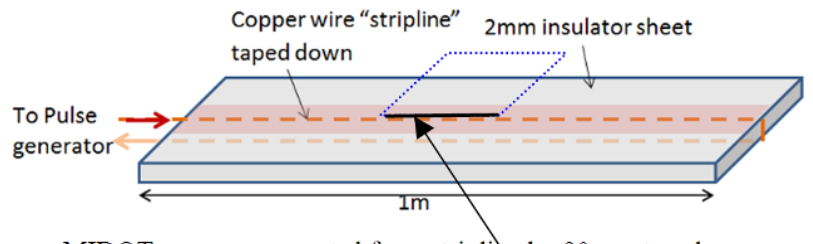

MIDOT sensor - separated from stripline by $90 \mu \mathrm{m}$ tape layer.

Figure 2, Pseudo-Stripline transmission line experiment; return path $2 \mathrm{~mm}$ directly below top conductor.

The use of the pseudo-stripline geometry immediately highlights the benefit over a single filament arrangement as shown in Figure 3. The mutual inductance values are obtained using standard expressions developed by Grover [5].

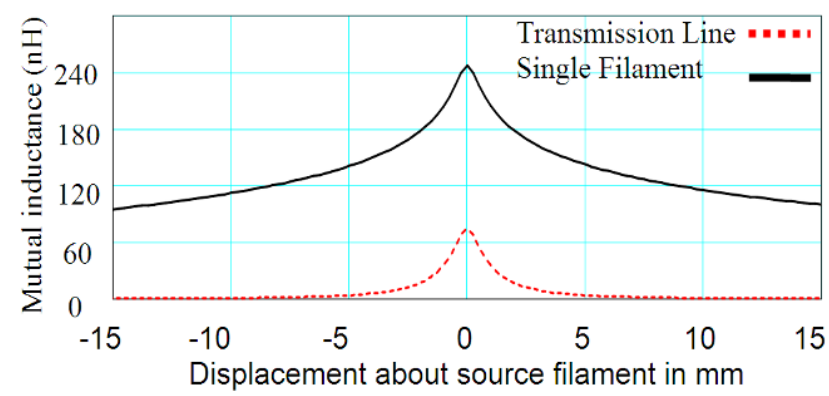

Figure 3, Modelled mutual coupling for a single source filament compared to a $2 \mathrm{~mm}$ separated stripline arrangement.

Despite a lower net signal, the transmission line arrangement gives a more localised mutual coupling to the sensor, which quickly drops to zero with lateral distance from the source current filaments. In this particular configuration the MIDOT is only sensitive to current flowing $\sim 2 \mathrm{~mm}$ either side of it. Whereas in the single filament arrangement, strong mutual coupling to the source current is observed even $15 \mathrm{~mm}$ away, making it much more difficult to distinguish between adjacent source filaments, therefore reducing the positional sensitivity of the sensor.

\section{Transmission line experiments}

When a $1 \mu \mathrm{s}, 0.5 \mathrm{~A}$ square current pulse is fed into a single filament of the pseudo-stripline, the induced voltage measured by a single MIDOT is shown in Figure 4. For detection of systematic error, two signals are measured during equivalent discharges. In each instance, the high impedance probe is connected to opposite ends of the MIDOT. These two orientations have been defined as the positive and negative polarity of the MIDOT.

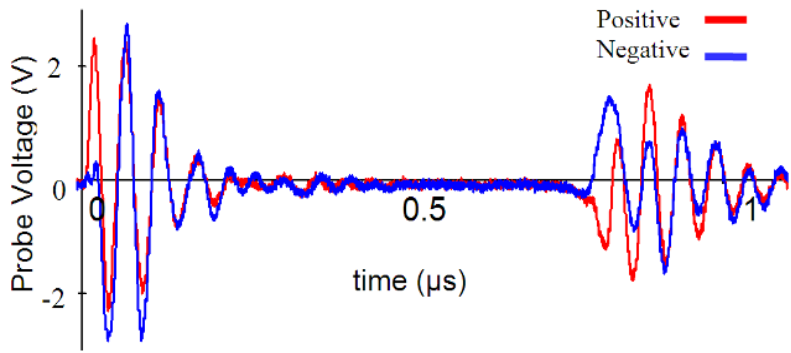

Figure 4, Raw MIDOT signals (positive and negative) from the stripline.

$$
V^{ \pm}{ }_{\text {MIDOT }}= \pm M d I / d t+\frac{Q}{C}+(\text { noise })
$$

Subtracting the 'negative' signal from the 'positive' and dividing by two removes any capacitive components as well as any noise and ringing due to the asymmetrical loading of the MIDOT circuit. This leads to a cleaner signal, as shown in Figure 5. This procedure is equivalent to using a high impedance differential probe.

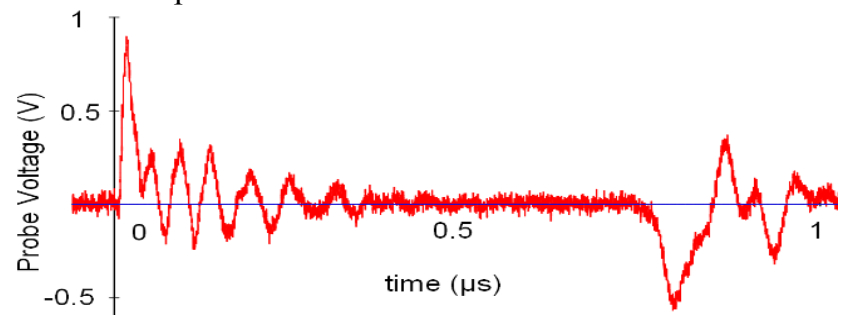

Figure 5, Processed MIDOT signal from the stripline arrangement.

Integrating the corrected signal shown in Figure 5. allows the sensor current to be measured which can be compared to the current in the known source filaments allowing the mutual inductance to be determined. The modelled and measured mutual inductance results for a range of lateral separations are shown in Figure 6.

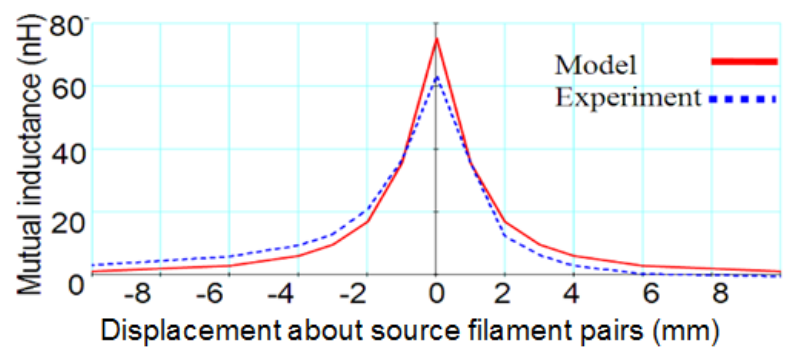

Figure 6, Experimental and modelled mutual inductance coupling for stripline geometry.

Figure 6. confirms the MIDOT performance with the transmission line geometry that was predicted in Figure 3. It also shows that it would be possible to position a series of MIDOTS 
3-4 $\mathrm{mm}$ apart to determine the current flow across a real continuous stripline in 3-4 mm strips.

\section{MIDOT ARRAY ASSEMBLY}

A Array development

An array of MIDOT sensors allows data from across a continuous stripline to be obtained without having to move the actual equipment, ensuring consistency between shots. Comparing the results from the single MIDOT scan and equivalent data from the array confirmed that the presence of multiple sensors does not affect the results. This is a consequence of the extremely low current $(\mu \mathrm{A})$ flowing through the sensors which are all terminated with high impedance voltage probes when in use.

The array was constructed by fixing 50 MIDOT filaments into channels machined in a polycarbonate sheet (the channels were $500 \mu \mathrm{m}$ wide and $500 \mu \mathrm{m}$ deep, with the adjacent channels spaced $2 \mathrm{~mm}$ apart) as seen in Figure 7.

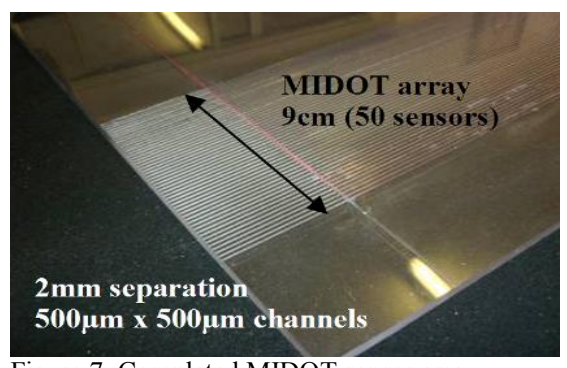

Figure 7, Completed MIDOT sensor array.

A schematic of the array is shown in Figure 8. The perpendicular sections of each loop run in a single (wider) channel. The sensor wire is enamelled which keeps all of the loops electrically isolated.

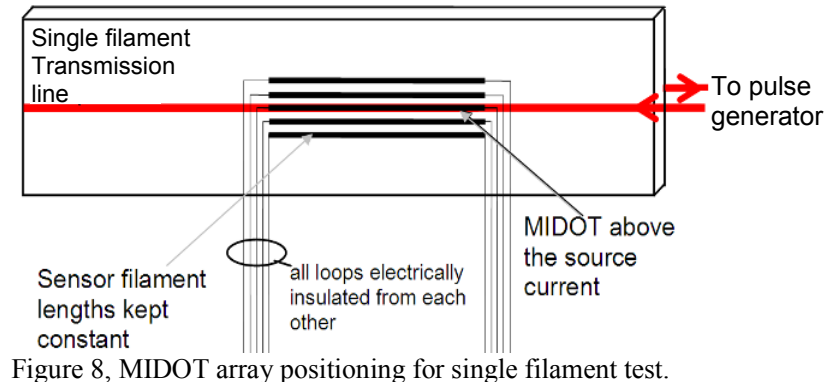

Figure 9. shows how the induced voltage measured on 2 adjacent sensors varies for the same source current using the MIDOT array.

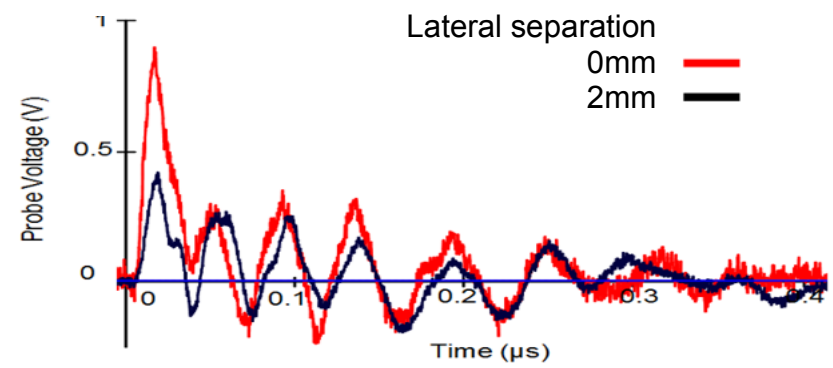

Figure 9, Processed MIDOT signals for ' $0 \mathrm{~mm}$ ' and ' $2 \mathrm{~mm}$ ' separation.

Integrating the above signals provides good agreement with Figure 6; proving there is no effect on the system due to the large number of parallel sensors. However it was found that using multiple MIDOT probes on the same oscilloscope did affect the readings, indicating that each MIDOT probe will need an independent data capture method.

The MIDOT sensor is also very sensitive to its height away from the source. As the depth of the machined channel is reduced, the separation between the MIDOT and the pair of go and return conductors for each pseudo-stripline reduces significantly, especially for the 'zero lateral displacement' position. A difference of even a few hundred microns in the depth of the MIDOT plane and the source filament plane can cause a noticeable variation in performance as shown in Figure 10 .

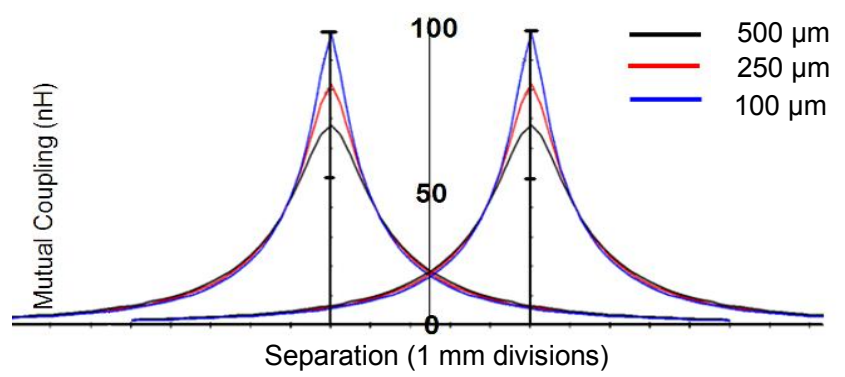

Figure 10, Modelled MIDOT coupling for three different channel depths.

As expected, the closer the sensor is to the current source, the stronger the coupling. Figure 10. also indicates that it should be possible to easily distinguish two separate current source filaments which are $4 \mathrm{~mm}$ apart using the machined array. This is yet to be proven.

For a given change in vertical separation at the zero position, the change in coupling for adjacent sensors is minimal. This is due to the MIDOT sensors being positioned $2 \mathrm{~mm}$ apart. For instance if the height is reduced from $500 \mu \mathrm{m}$ to $250 \mu \mathrm{m}$, the zero position separation will decrease by $50 \%$, whereas the diagonal separation to the next adjacent sensor only reduces by $\sim 3 \%$. This geometric feature is reflected in the mutual inductance coupling of the sensors to active pseudo-stripline filaments. 


\section{B MIDOT calibration}

Passing a known current pulse under each sensor in the array and recording the induced voltage allows the array to be calibrated i.e. determine the actual mutual inductance for each MIDOT sensor in the array. An error of $\pm 10 \mathrm{nH}( \pm 15 \%)$ in the mutual coupling of the MIDOT to the source filament is found due to errors in the vertical positioning of the MIDOT during construction. These can be removed in the future by using a more controlled manufacturing process such as laser etching or a PCB manufacturing technique.

\section{$C$ Multiple filament tests}

The following tests were designed to assess the capability of the array to resolve multiple current sources. The $9 \mathrm{~cm}$ wide pseudostripline was represented by 17 individual pairs of filaments, with the upper conductor shown in Figure 11. These are spaced $5 \mathrm{~mm}$ apart and can be independently connected to a Stanford pulse generator to allow any combination of these filaments to be activated as necessary.

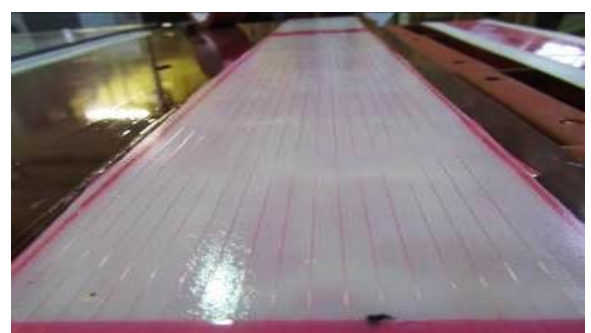

Figure 11, Filamentary analogue of flat transmission line.

A schematic outlining how two of these filaments can be simultaneously activated is shown in Figure 12.

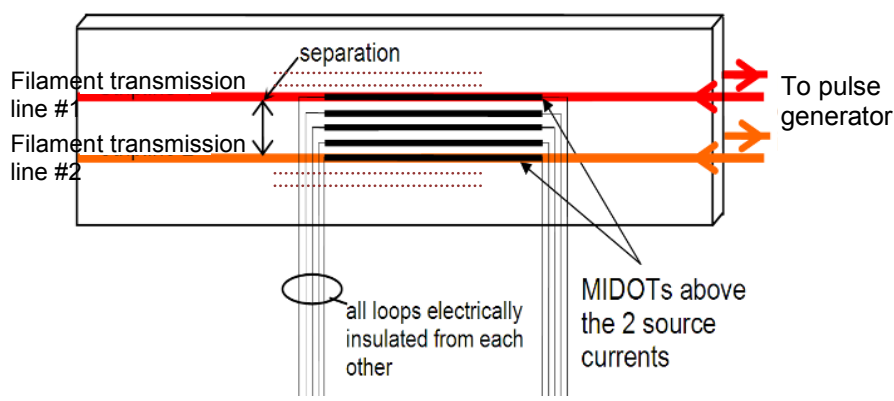

Figure 12, Dual source filament pairs test schematic.

All tests to date have utilised two source filament pairs as indicated in Figure 12. The separation between the two source filament pairs was varied from $40 \mathrm{~mm}$ to $5 \mathrm{~mm}$. The results from these experiments are shown below.

Figure 13. shows the results for the $40 \mathrm{~mm}$ separated source experiments. The signal from filaments 20 and 40 are clearly the most dominant and therefore we can use this to locate the source currents.

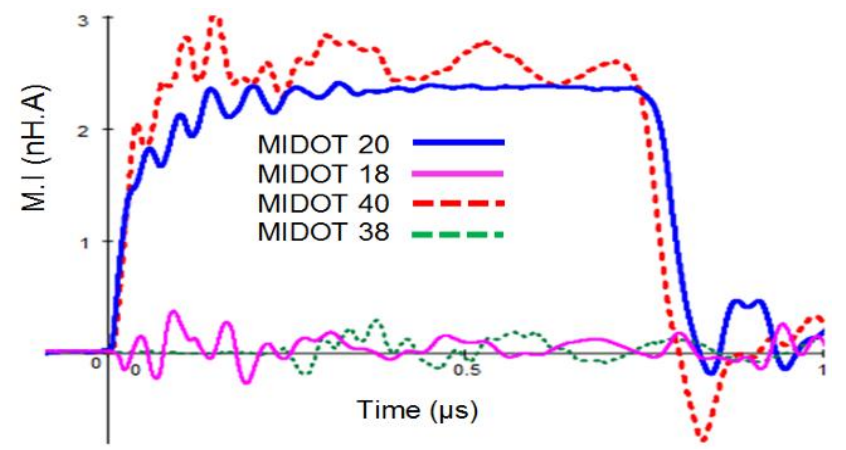

Figure 13, Processed MIDOT data, MIDOTs 20 and 40 clearly identified as closest to the source filaments.

Similar experiments with varying separations were conducted with results shown in Figures 14-16.

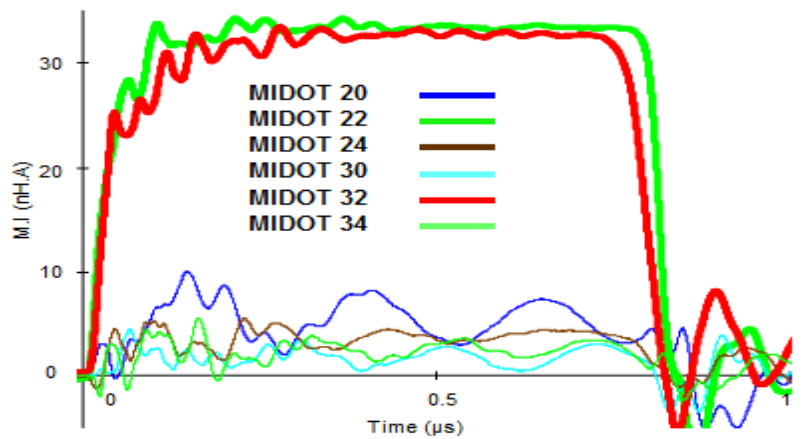

Figure 14, Processed MIDOT data, MIDOTs 22 and 32 clearly identified as closest to the source filaments.

Figure 14. shows that the two source filaments are $20 \mathrm{~mm}$ apart and can be easily distinguished from the rest of the signals.

For a $10 \mathrm{~mm}$ separation the results are given in Figure 15.

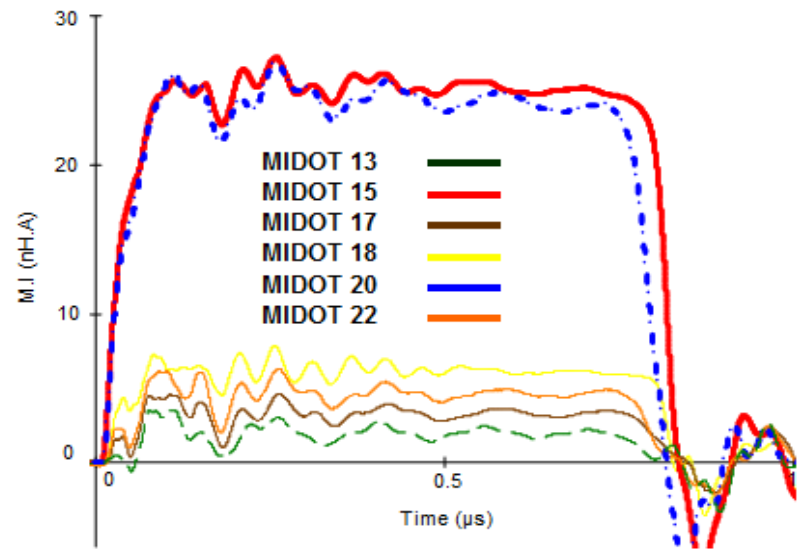

Figure 15, Processed MIDOT data, MIDOTs 15 and 20 clearly identified as closest to the source filaments. 
The peak signals from the $5 \mathrm{~mm}$ separated arrangement shown in Figure 16. still allow current source locations to be easily identified.

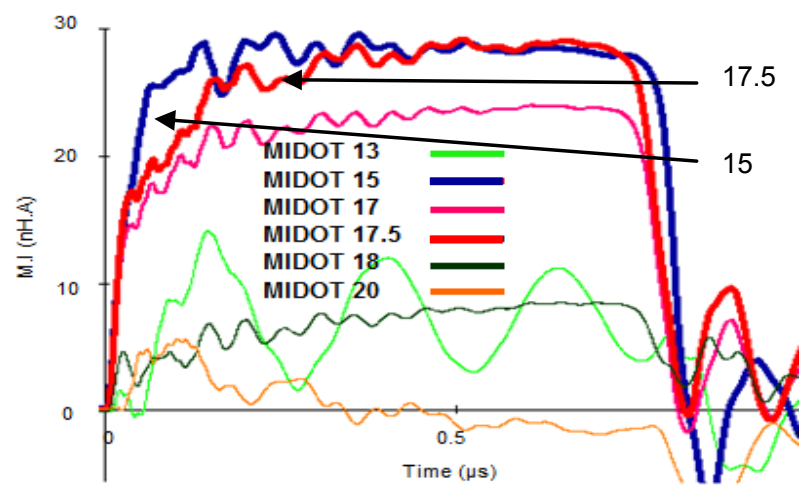

Figure 16, Processed MIDOT data, MIDOTs 15 and17.5 clearly identified as closest to the source filaments

In this arrangement, the peak signal occurred in MIDOT 15, however the second peak signal was difficult to find and it was determined to lie between MIDOT 17 and 18. This was confirmed by moving the sensor array to have a sensor above the identified region and this allowed the "MIDOT 17.5" signal to be captured. The data in Figure 16. allows the location of the two source filaments to be accurately determined, and this arrangement also confirms that the MIDOT array is capable of distinguishing source filaments which are $5 \mathrm{~mm}$ apart.

Figure 17. shows the actual current levels determined during the sensor calibration. Errors in the lateral positioning of the MIDOT sensors allow MIDOT( $\min )$ and $\operatorname{MIDOT}(\max )$ to be obtained.

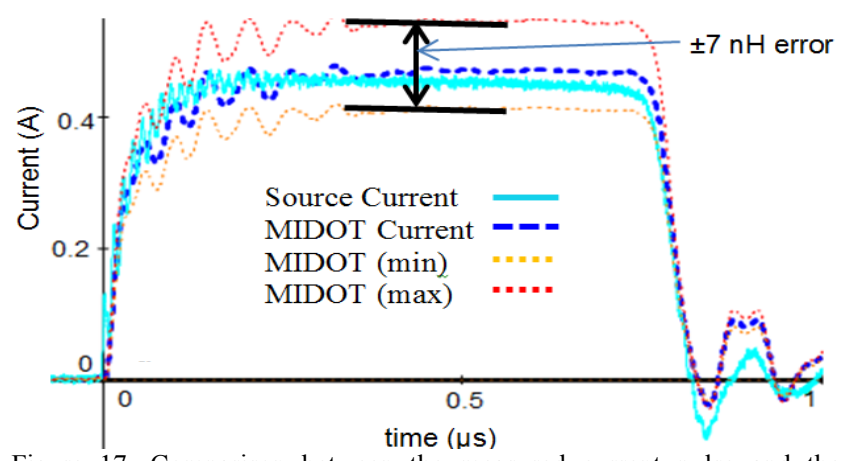

Figure 17, Comparison between the measured current pulse and the current determined from MIDOT 20.

When using the theoretically calculated mutual inductance, the MIDOT current is in very good agreement with the measured source current. It is clear to see that the MIDOT technique can determine the location of and also the absolute value of independent source current filaments in the low current experiments.

It is also possible to plot a relative current distribution across the array as shown in Figure 18.

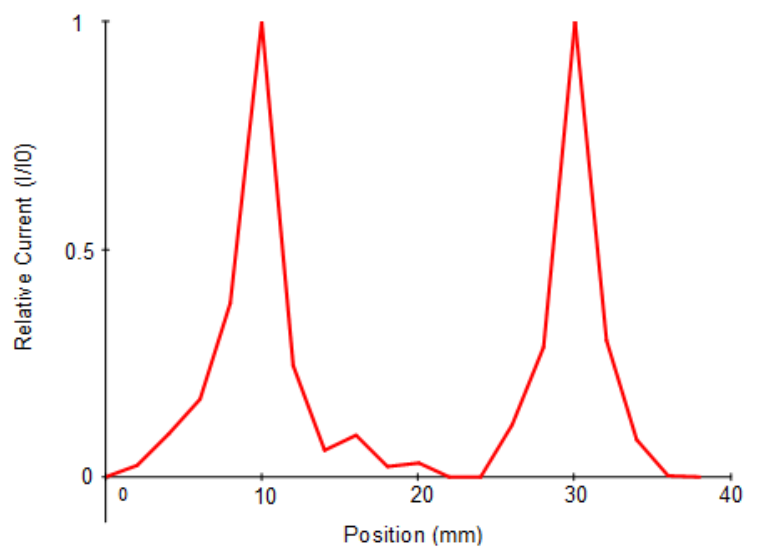

Figure 18, Relative current distribution across a $4 \mathrm{~cm}$ section of the stripline.

The two source filaments are clearly seen to be $2 \mathrm{~cm}$ apart and if the conductor was assumed to be a continuous sheet, Figure 17. would be indicative of the current distribution across the stripline.

\section{$D$ Competing techniques described in the literature and the advantage of using MIDOT arrays.}

Techniques to determine the current distributions have been published previously [6]; one of the more recent approaches has been to use the Colossal Magneto Resistive (CMR) effect of specific materials [7]. The major drawback of this technique is that a complex computer model is necessary to determine the current distribution, required to generate the magnetic field causing the measured effect. It is also possible for a number of different current distributions to generate the same total magnetic field at a particular location. Another important factor that cannot be neglected is the cost of the probes. A single CMR probe is much more expensive than all the filaments utilised in a MIDOT array!

The benefit of the MIDOT approach is that a direct and localised measurement of the current (or immediate effect of the presence of current) is obtained.

Due to the design of the MIDOT sensor, it automatically integrates the measurement along the whole length of the sensor. This in effect reduces the problem to 2D. The CMR probes provide sufficient localisation but are only $500 \mu \mathrm{m}$ long and so require a large number of sensors positioned along the length of the flyer during a single shot to obtain data equivalent to that obtained from a MIDOT array during a single shot. 


\section{E Initial high current tests}

Experiments were carried out on the QUATTRO bank at Loughborough [7] using 2 MIDOT filaments which were $50 \mu \mathrm{m}$ thick and $2.5 \mathrm{~mm}$ wide (the closest match to the filament size in the 2D model described in [1]) as shown in Figure 19.

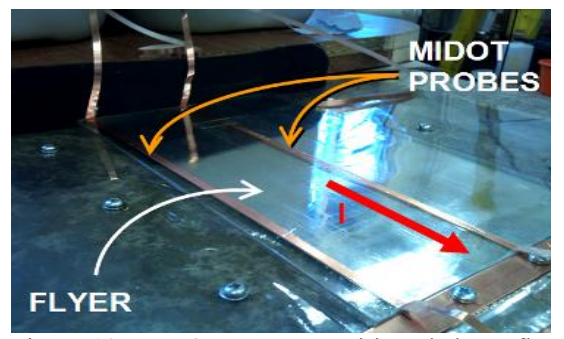

Figure 19, MIDOT sensors positioned above flyer plate section on Quattro.

Placing one sensor in the centre of the flyer plate and another along the edge allowed the largest difference in signals to be measured. Figure 20. shows the total current pulse during a QUATTRO discharge. The associated MIDOT, induced voltage data compared with prediction from the $2 \mathrm{D}$ model is shown in Figure 21.

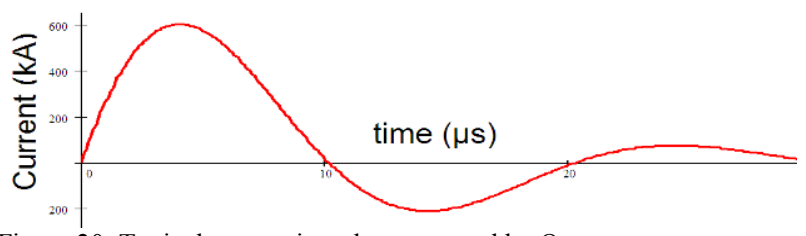

Figure 20, Typical current impulse generated by Quattro.

Figure 21. is a comparison of the modelled and measured voltage for the probe at the edge of the flyer.

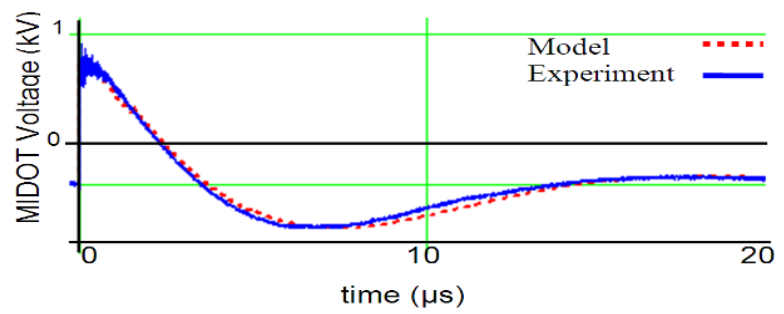

Figure 21,2D theoretical induced voltage predictions compared with experimental data for a MIDOT placed $250 \mu \mathrm{m}$ above the flyer.

The results from these experiments show that a MIDOT sensor array generates data that can be used to better understand the transient, non-uniform current flow in a parallel plate transmission line. A large series of tests using a dedicated experimental test-bed are about to be conducted to further develop the understanding of the MIDOT array sensor fielded in high current systems.

\section{CONCLUSIONS AND THE WAY AHEAD}

A novel type of sensor termed MIDOT has been invented, designed, manufactured and successfully tested on both low and high current systems. A MIDOT sensor, which only uses straight, very thin wires, can produce an open circuit signal which can be integrated to reveal the current in an adjacent conductor. Even more, the MIDOT appears to be sufficiently sensitive to be able to identify the lateral position of a pair of transmission line filamentary conductors with a precision better than $\pm 2 \mathrm{~mm}$. Using these two properties, a MIDOT array can be used to determine the time-variation of the current distribution in a parallel-plate transmission line.

Further work is already planned to be conducted on the MIDOT technique using a specially constructed high-current test-bed. One benefit of moving to a higher current will be that the noise and capacitive stray effects associated with the low current results presented in this paper will be much reduced so that the analysis will become more accurate.

Finally, MIDOT arrays will be used on the the QUATTRO facility to probe the time varying current distribution during a flying plate shot.

\section{REFERENCES}

[1] K. Omar et al, "FOIL-FLYER Electro-Magnetic Accelerator, Initial Results From A New AWE Pulsed Power Generator," 18th IEEE Int. Pulsed Power Conference, Chicago, IL, USA. 2011.

[2] B. Novac, et al, "Numerical modelling of a flyer plate Electromagnetic Accelerator," IEEE Trans. on Plasma Science, 40(10), pp. 2300-2311, Oct 2012.

[3] C. Mielke and B.M. Novac, "Experimental and Numerical Studies of Megagauss Magnetic Field Generation at LANL-NHMFL," IEEE Trans on Plasma Science, 38(8), pp. 1739-1749, 2010.

[4] K. Omar et al, "Flyer Plate Electromagnetic Accelerator Modelling and Development of Bespoke Sensor Technology," presented at 14th Int. Conf. Megagauss Magnetic Field Generation and Related Topics, Maui, Hawaii, 2012.

[5] F.W. Grover, Inductance Calculations. Working Formulas and Tables, Dover Publications, Inc., New York, 1946.

[6] O, Leibfried et al, "Measurement of the Magnetic field Distribution in railguns using CMR-B Scalar sensors," in proc. ACTA physica Polonica A, Vol 115. (2009), pp1125-1127.

[7] S. Balevicius et el, "High frequency CMR-B Scalar sensor for Pulsed Magnetic field Measurements," 4th EAPPC, Karlsruhe, Germany, 2012 